\title{
Construções sustentáveis na Engenharia Civil e a responsabilidade socioambiental
}

\section{Neucy Teixeira Queiroz}

Universidade Federal de Minas Gerais (UFMG). Av. Universitária, 1000. Bairro Universitário. Montes Claros-MG (CEP 39404-547). E-mail: neucyqueiroz@yahoo.com.br.

Resumo. Este trabalho busca explorar a ideia de construção sustentável no espaço urbano, pois, à medida que a população cresce e as cidades se desenvolvem, o campo da construção civil cresce gradativamente e, em consequência, ocorre a expansão de escolas, hospitais, aeroportos, etc. A Engenharia Civil tem gerado impactos ambientais que podem ser minimizados e resolvidos. Por isso, é preciso buscar uma relação harmônica com o meio ambiente, uma vez que é necessário um equilíbrio ambiental para que haja um desenvolvimento que beneficie a sociedade atual, sem que ocorra esgotamento dos recursos naturais. A utilização de materiais sustentáveis nas construções e a aplicação dos princípios da sustentabilidade têm demonstrado alternativas para o desenvolvimento sustentável na área de construção civil. Preocupações no setor econômico, social e ambiental, devem ser levadas em consideração visando à qualidade de vida da sociedade, contribuindo para a sustentabilidade do meio ambiente. Apesar de haver degradação ambiental na construção civil, é possível melhorar o vínculo seu com o meio ambiente, pois as construções são necessárias e importantes no desenvolvimento das cidades. As construções sustentáveis representam uma ideia que pode minimizar os problemas ambientais, contribuindo para a sustentabilidade. O presente artigo analisa a aplicação de construções sustentáveis na Engenharia Civil e de que forma a responsabilidade socioambiental se manifesta. Para a realização desse trabalho, foi utilizada como metodologia a pesquisa bibliográfica e foi possível perceber que há pouco uso de construções sustentáveis. Entretanto, é possível ampliar essa ideia nos cursos voltados para a construção civil, pois construções sustentáveis demonstram preocupação socioambiental o que é fundamental para o desenvolvimento sustentável.

Palavras-chave: Construção; Sustentabilidade; Responsabilidade socioambiental.

Abstract. Sustainable constructions in Civil Engineering and the
social-environmental responsibility. The present work seeks to
explore the idea of sustainable construction in urban areas, since, as
the population grows and cities develop, the construction field also
graduall y grows too, and, as a consequence, occurs the expansion of
schools, hospitals, airports, etc. Civil Engineering has generated
environmental impacts that can be minimized and resolved.
Therefore, we must seek a harmonious relationship with the
Recebido:

04/07/2016

Aceito:

03/09/2016

Publicado:

$31 / 12 / 2016$

Acesso Aberto

Artigo completo

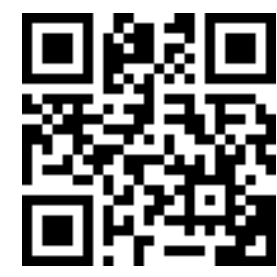

ORCID

(1) 0000-0002-7387-1566

Neucy Teixeira Queiroz 
environment, since an environmental balance is necessary so that there is a development which benefits the current society, without depletion of natural resources. The use of sustainable materials in the constructions and the application of sustainability principles, have demonstrated alternatives for sustainable development in the civil construction field. Concerns in the economic, social and environmental sectors, must be taken into consideration aiming at the society's quality of life, contributing to environmental sustainability. Although there is environmental degradation in construction, it is possible to improve the bond of civil engineering with the environment, as buildings are necessary and important in the development of cities. The sustainable constructions represent an idea that can minimize environmental problems, contributing to sustainability. This article analyzes the application of sustainable constructions in Civil Engineering and in what way environmental responsibility is manifested. To perform this work, a bibliographic research was used as methodology, and it was possible to perceive that there is little use of sustainable constructions. However, it is possible to extend this idea on the courses designed for civil construction, as sustainable constructions demonstrate a socialenvironmental concern, which is fundamental for sustainable development.

Keywords: Construction; Sustainability; Social-environmental responsibility.

\section{Introdução}

As cidades estão em constante desenvolvimento, expandindo-se cada vez mais. Com o crescimento da população no meio urbano, faz-se necessário a ampliação de indústrias, hospitais, escolas, aeroportos, e etc. Sendo assim, é necessário um planejamento no uso desses espaços. E nesse momento assoma-se a engenharia civil. A atuação desse setor tem papel fundamental no processo de urbanização; não obstante, envolve um processo complexo, já que a estruturação dos projetos passa por várias etapas, a fim de organizar o espaço a ser construído. Apesar de a construção civil ser o ponto de partida para o desenvolvimento das cidades e para a organização espacial, ela gera algum tipo de poluição. Mendonça (2015) evidencia que a poluição se manifesta de várias formas, dentre as quais se destacam a atmosférica e a da água. De acordo com o Programa de Meio Ambiente das Nações Unidas (UNEP, 2007), 30\% a $40 \%$ das emissões de gases, com efeito de estufa, devem-se aos edifícios e à indústria da construção, incluindo a produção de materiais de construção, manutenção e demolição, e a fase de uso.

Tudo isso exige uma séria preocupação ambiental, uma vez que os gases de efeito estufa estão relacionados com o aumento da temperatura, contribuindo assim, para o aquecimento global que vem sendo um problema bastante crítico na atualidade, principalmente nas chamadas "ilhas de calor”, presentes nos grandes centros urbanos. A poluição pode ainda contribuir para gerar outros desequilíbrios ambientais, sendo necessários critérios que visem à sustentabilidade na engenharia civil. $\mathrm{O}$ uso de materiais sustentáveis nas construções tem sido uma alternativa interessante, pois visa a contribuição ambiental. Além disso, devem-se focalizar construções com a permanência do maior número possível de árvores, pois elas exercem papel na redução do aquecimento global, já que fixa o gás carbônico $\left(\mathrm{CO}_{2}\right)$ que é responsável pelo aumento da temperatura do planeta. 
O Presente trabalho teve como objetivo analisar a aplicação de construções sustentáveis na engenharia civil e verificar de que forma a responsabilidade socioambiental se manifesta. Para isso, foi utilizada como metodologia a pesquisa bibliográfica.

\section{O conceito de desenvolvimento sustentável}

O conceito de desenvolvimento sustentável não é muito antigo. Foi definido pela Comissão Mundial Sobre Meio Ambiente e Desenvolvimento (1987) como aquele que permite satisfazer as necessidades do presente sem comprometer as possibilidades das gerações futuras satisfazerem as suas. Tendo em vista essa definição, pode-se deduzir que o desenvolvimento sustentável só é possível se houver uma preocupação com a conservação dos recursos naturais, pois a sociedade é completamente dependente deles para a sua sobrevivência, por isso, faz-se necessário estabelecer uma relação de equilíbrio socioambiental.

A construção civil merece uma atenção enorme em se tratando de desenvolvimento sustentável, pois é fator fundamental para avanço da sociedade. A urbanização deve sempre priorizar a preocupação com o meio ambiente. Os recursos hídricos, por exemplo, são fundamentais à sobrevivência humana, pois estão relacionados com geração de energia, produção agropecuária, desenvolvimento industrial, doméstico, etc. Por isso, deve haver planejamento nas formas de uso da água nos projetos de construção como, por exemplo, meios possíveis de estocar água de chuva. Deve-se atentar também ao uso materiais de isolamento acústico, a fim de proporcionar tranquilidade às pessoas que residem próximo e aos que habitarão aquele imóvel a ser construído. Outro fator importante, que demonstra preocupação socioambiental, é proporcionar ambientes que tenhamvegetações, pois elas melhoram a qualidade do ambiente, proporcionando frescor.

\section{Construções sustentáveis}

A Engenharia Civil precisa buscar meios que não causem danos ao meio ambiente, ou caso causem, que sejam os mínimos possíveis. Quanto aos danos ambientais, é possível encontrar alternativas que possam melhorar a construção no sentido de torná-la mais sustentável.

Couto et al. (2006) apontam que o uso de materiais reciclados pode ser uma das alternativas para uma construção mais sustentável. "O uso de materiais reciclados irá encorajar indústrias e governos a investigar novas tecnologias para reciclar, e para criar uma rede de suporte mais larga para futura reciclagem e reutilização" (Couto et al., 2006). O uso de materiais sustentáveis evidencia maior responsabilidade com o meio ambiente, sendo interessante ampliar esse tipo de construção. Sobre os materiais sustentáveis, Wieczynski (2015), destaca o tijolo ecológico, as placas ecológicas, captação de água de chuva e ressalta a importância ecológica desses materiais: quanto ao tijolo, é ecológico porque, diferentemente do tijolo convencional, não precisa ser cozido em fornos, eliminando assim a utilização de lenha e a derrubada de dez árvores para a fabricação de mil tijolos. Sendo assim, sem a lenha ficamos sem fumaça, sem emissão de gases de efeito estufa; as placas ecológicas são feitas com material 100\% reciclado pós-consumo, há baixa absorção de umidade $(<4 \%)$, é resistente a agentes químicos, é isolante termo-acústico e a captação de água de chuva colabora para reduzir o valor da conta de água, serve de reserva em períodos de seca ou falta d'água, reduz a necessidade de água para fins não potáveis no imóvel, como regas de jardins, lavagem de automóveis e descarga de vasos sanitários,limpezas domésticas e educa ambientalmente quem tem contato com o sistema.

A engenharia civil tem uma relação ambiental e social, já que contribui também para geração de emprego e ajuda a circular a economia de um país. Sendo assim, é necessária uma preocupação ainda mais acentuada para obter construções 
sustentáveis. Sobre economia, emprego e impactos ambientais, Pinheiro (2003) mostra que:

\begin{abstract}
A indústria da construção, respondendo às necessidades sociais e económicas, cria e implanta infra-estruturas (estradas, barragens, linhas de caminho de ferro), zonas urbanas (Edifícios e Parques), promovendo o crescimento (representava na Europa em 1999 OCDE, 2003 - 9,7\% do Produto Nacional Bruto e $7,5 \%$ do emprego) e suporte os processos de desenvolvimento. As actividades da sua tipologia induzem também, em muitos casos, alterações substanciais no ambiente, incluindo impactes ambientais muito significativos. Em muitos casos, esses impactes, ou pelo menos parte deles, são de carácter negativo e afectam decisivamente o ambiente actual e futuro (Pinheiro, 2003).
\end{abstract}

Os impactos ambientais exercem extrema importância na construção civil,pois seus efeitos podem comprometer o desenvolvimento sustentável. Além de conhecer o espaço onde haverá a construção, é necessário escolher de forma cautelosa os materiais a serem utilizados, pois, eles podem causar impactos ambientais catastróficos. Para se perceber a importância dos materiais de construção no contexto da construção sustentável, importa saber desde logo quais os impactos ambientais provocados pela extracção das matérias-primas necessárias à sua produção (Torgal e Jalali, 2010). É importante ainda envolver a atuação de profissionais diversos: engenheiros, arquitetos, ambientalistas, engenheiros florestais. $\mathrm{Ou}$ seja, a participação de profissionais da área ambiental contribui bastante no que se refere ao uso dos recursos naturais.

\section{Princípios da sustentabilidade}

Para considerar uma construção sustentável, é necessário levar em consideração alguns princípios a serem adotados nas construções civis. Para essa análise, consideram-se aqueles mencionados por Kibert (1994):

1. Minimizar o consumo de recursos;

2. Maximizar a reutilização dos recursos;

3. Utilizar recursos renováveis e recicláveis;

4. Proteger o ambiente natural;

5. Criar um ambiente saudável e não tóxico;

6. Fomentar a qualidade ao criar o ambiente construído.

As construções sustentáveis obedecem a esses princípios, pois sua finalidade é contribuir com o desenvolvimento sustentável. A minimização do consumo de recursos naturais possibilita sua preservação. A água, por exemplo, precisa ser economizada, sendo necessárias estratégias que propiciem a sua reutilização, pois a sua falta afetaria diretamente a vida no planeta. A proteção ambiental inclui a conservação da vida vegetal, uma vez que as plantas são responsáveis pelo equilíbrio da temperatura, ajudando a reduzir as concentrações de gás carbônico, contribuindo para a proteção do ambiente natural. Portanto, quanto menos madeira se utilizar em uma construção, maior será essa proteção. Também é importante criar um ambiente agradável, com maior frescor natural. Janelas grandes, por exemplo, possibilitam maior entrada de ar, e isso deve ser levado em conta no processo de construção.

Quando se opta pela utilizaçãode recursos renováveis e recicláveis nas construções, ocorre contribuição ambiental, na medida em que os recursos naturais são limitados eo uso indiscriminado desses recursos pode comprometer as gerações futuras a terem acesso aos bens naturais.

Para apontar uma construção como sustentável, Mateus e Bragança (2004), consideram algumas variáveis para avaliar a sustentabilidade. Esses parâmetros estão representados na Tabela 1. 
Tabela 1. Variáveis que podem ser considerados na avaliação da sustentabilidade.

\begin{tabular}{|c|c|c|}
\hline \multicolumn{3}{|c|}{ Indicadores } \\
\hline Ambiental & Funcional & Econômico \\
\hline Potencial de aquecimento global & $\begin{array}{l}\text { Isolamento sonoro a sons de } \\
\text { condução aérea }\end{array}$ & Valor de construção \\
\hline Energia primária incorporada & $\begin{array}{l}\text { Isolamento sonoro a sons de } \\
\text { percussão }\end{array}$ & Custo de manutenção \\
\hline Conteúdo reciclado & Isolamento térmico & Custo de reabilitação \\
\hline Potencial de reciclagem & Durabilidade & $\begin{array}{l}\text { Custo } \\
\text { desmantelamento/demolição }\end{array}$ \\
\hline $\begin{array}{l}\text { Reservas remanescentes de } \\
\text { matéria prima }\end{array}$ & Comportamento ao fogo & Valor residual \\
\hline $\begin{array}{l}\text { Quantidade de matéria/recursos } \\
\text { naturais utilizados }\end{array}$ & Flexibilidade de utilização & $\begin{array}{l}\text { Custo de tratamento para } \\
\text { devolução ao ambiente natural }\end{array}$ \\
\hline
\end{tabular}

Fonte: Mateus e Bragança (2004).

\section{Soluções sustentáveis para a construção \\ Com base na Tabela 1, proposta por} Mateus e Bragança (2004), pode-se incorporar soluções sustentáveis para a construção levando-se em conta os indicadores ambiental, funcional e econômico.

No aspecto ambiental, consideramse algumas soluções sustentáveis como maior atenção ao potencial de aquecimento global e de reciclagem, quantidade de matéria e recursos naturais utilizados de forma minimizada e a incorporação de energia primária.

No setor funcional, leva-se em conta o funcionamento do imóvel a ser construído, como isolamento térmico, sonoro, durabilidade, dentre outros.

No que se refere ao fator econômico, deve atentar-se aos procedimentos adotados na construção e aos custos, como por exemplo: custos de manutenção, construção, reabilitação. Uma das medidas que se pode tomar nesse aspecto é o uso de materiais sustentáveis na engenharia civil, o que reduz bastante os custos e colabora com a conservação dos recursos naturais. Contudo, devem-se procurar materiais que são viáveis à construção a ser feita, para não causar prejuízos à construção.

\section{Sustentabilidade e economia}

Os fatores econômicos devem ser levados em consideração durante a elaboração do projeto de construção civil, visando à sustentabilidade ambiental. Kibert (1994), diz que a Construção Sustentável tem como objetivo a criação e manutenção responsáveis de um ambiente construído saudável, baseado na utilização eficiente de recursos e em princípios ecológicos.

Economia e sustentabilidade caminham lado a lado no processo de desenvolvimento. Alguns fatores são apontados por Silva Mateus (2009), para evidenciar maneiras que contribuem para o desenvolvimento sustentável na construção civil, enfatizando a relação economia e sustentabilidade. Economizar os recursos renováveis, utilizar materiais reutilizáveis ou descartáveis, visando sempre à economia e qualidade. Silva Mateus (2009) resume na Figura 1 as prioridades que são necessárias, na construção de um projeto de construção sustentável. 


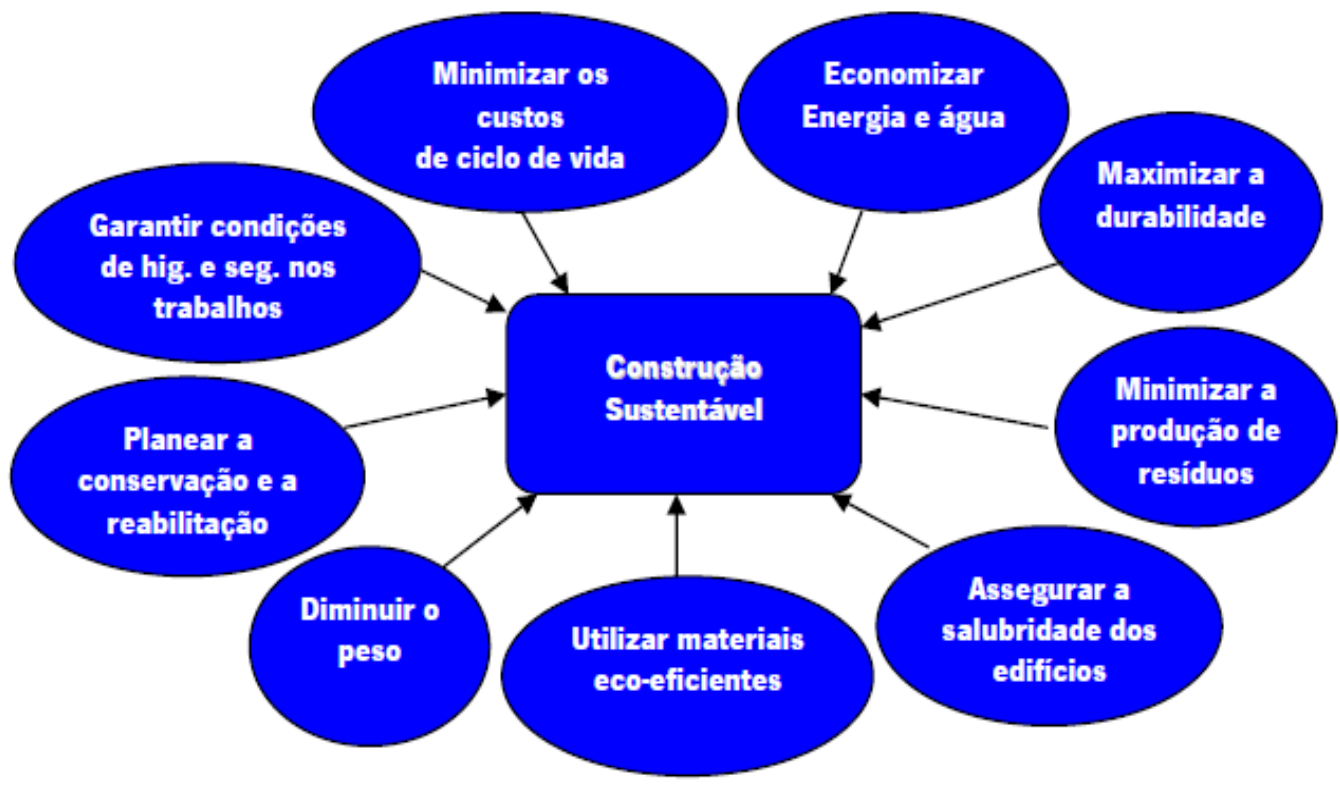

Figura 1. Resumo das prioridades a se adotar no projeto de uma construção sustentável. Fonte: Silva Mateus (2009).

Quanto às prioridades mostradas na Figura 1 em projetos de construções civis, todas elas se relacionam com a economia, já que, entre outras, a minimização da produção de resíduos sólidos, a economia de energia e água e a maximização da durabilidade podem contribuir para reduzir custos.

Solano (2008) aponta que, quando não é possível recusar um produto ou material, há a possibilidade de reduzir o consumo do mesmo. Reduzindo sua quantidade, reduz também o lixo gerado por ele, seja pelas embalagens ou pelo descarte em si. Na construção civil pode-se, por exemplo, reduzir a quebra de tijolos solicitando ao fornecedor de blocos cerâmicos o envio de blocos previamente cortados.

Economizar energia é importantíssimo para uma construção sustentável. De acordo com Rocheta e Farinha (2007), na Europa os edifícios constituem o espaço onde as pessoas permanecem cerca de $90 \%$ do seu tempo, pelo que uma inadequada gestão energética contribui para elevados gastos de energia em climatização. Compreende-se, assim, a importância da implementação de práticas de projecto e construtivas que reduzam os gastos energéticos e recorram a formas de energia renováveis.

Corrêa (2009) explicita que é interessante utilizar o clima de forma favorável à construção. Como um mesmo município pode possuir zonas climáticas distintas, estratégias diferenciadas devem ser utilizadas na implantação de edificações, para permitir o aproveitamento das potencialidades microclimática e subseqüente bom desempenho energético, reduzindo-se o uso de aparelhos como ventiladores ou climatizadores de ar, gerando assim, maior economia.

A utilização dos sistemas naturais de controle térmico (insolação, ventilação) deve ser explorada ao máximo e os mecanismos artificiais devem ser eficientes (Cianciardi e Bruna, 2004).

Rocheta e Farinha (2007) mostram atenção quanto aeconomia de água nas edificações e apontam o aproveitamento das 
águas de chuvas como uma solução viável na economia dos recursos hídricos:

\begin{abstract}
As águas pluviais podem ser utilizadas para regas, lavagens bem como para o abastecimento de autoclismos de sanitas. Não podem ser usadas para preparação de alimentos nem para duches, pois existe o risco de presença de bactérias. Os sistemas de aproveitamento destas águas incorporam um tanque de armazenamento, um sistema de filtros e outro de distribuição com bomba e tubagens. Para um rentável aproveitamento é necessário que o edifício disponha de uma área de cobertura significativa.
\end{abstract}

O tempo de vida das construções está intimamente relacionado com a economia no âmbito da engenharia civil.

Para John et al. (2002), o planejamento da vida útil introduz a previsão da vida útil na fase de projeto, incluindo as etapas da definição da vida útil de projeto do edifício, da previsão da vida útil estimada dos componentes do edifício, bem como do emprego da vida útil estimada como critério para seleção, ainda durante a fase de projeto, da alternativa que minimize o custo global.

É por essa razão que John et al. (2002) advogam que a durabilidade, expressa pela distribuição de vida útil de um conjunto de componentes, desempenha uma função importante para a obtenção de uma construção sustentável.

A gestão de resíduos sólidos se enquadra nas atividades de saneamento básico, pois existe a interdependência entre este, a saúde e o meio ambiente. Portanto, as ações de gerenciamento de resíduos da construção civil devem ser interrelacionadas para contribuir com a melhoria da qualidade ambiental proporcionada à população (Mariano, 2008).

Para que a construção seja sustentável e viável economicamente é preciso executar a obra procurando obedecer aos princípios de sustentabilidade, buscando sempre a preocupação socioambiental.

\section{Considerações finais}

Embora a Engenharia Civil seja necessária para o desenvolvimento das cidades, ela pode provocar danos à natureza, se não houver relação de equilíbrio entre construção e meio ambiente. Tal equilíbrio pode ser obtido por meio de um planejamento que leve em consideração as diversas proposições inerentes aos princípios de sustentabilidade, e que resultem em construções sustentáveis. Um planejamento socioambiental satisfatório no ambiente urbano minimiza os impactos ambientais.

Com a evolução do conhecimento, evidenciam-se as construções sustentáveis que são importantíssimas para os projetos de construções, pois, contribuem para maior preservação dos recursos naturais. Apesar do surgimento dessa ideia transformadora, há carência na expansão desse tipo de construção. Um maior incentivo nos cursos de graduação, juntamente com a ampliação do conhecimento acadêmico acerca do assunto, pode contribuir para a formação de um engenheiro mais consciente, no que se refere à preservação ambiental; pois esta reflete diretamente na sociedade. $\mathrm{O}$ foco voltado para minimização dos impactos ambientais no ensino da engenharia civil pode trazer ideias ainda mais inovadoras acerca dessa temática.

A utilização de materiais sustentáveis nas construções e os princípios de sustentabilidade têm-se constituído como alternativas bem viáveis, mas é possível ampliar essas ações e colocá-la ainda mais em prática. Afinal, desequilíbrios ambientais podem ter consequências graves como falta de água, aumento do calor, maior poluição, dentre outras. Uma maior conscientização no ensino da engenharia, nessa perspectiva, pode contribuir para uma maior preocupação socioambiental, auxiliando o desenvolvimento sustentável.

\section{Declaração de conflito de interesses}

A autora declara não haver conflito de interesses. 


\section{Referências}

Cianciardi, G.; Bruna, G. C. Procedimentos de sustentabilidade ecológicos na restauração dos edifícios citadinos. Cadernos de PósGraduação em Arquitetura e Urbanismo, v. 4, n. 1, p. 113-127, 2004. Disponível em: $<$ http://coral.ufsm.br/lasac/patrimonio5.pdf>.

Acesso em: 22 ago. 2016.

Comissão Mundial Sobre Meio Ambiente e Desenvolvimento. Nosso Futuro Comum. 2 ed. Rio de Janeiro: FGV, 1991.

Corrêa, L. R. Sustentabilidade na construção civil. Belo Horizonte: Escola de Engenharia, UFMG, 2009. (Monografia de graduação). Disponível em: <http://especializacaocivil. demc.ufmg.br/trabalhos/pg1/Sustentabilidade na Construção CivilL.pdf>. Acesso em: 22 ago. 2016.

Couto, A. B.; Couto, J. P.; Teixeira, J. C. Desconstrução: uma ferramenta para a sustentabilidade da construção. Anais do VI Seminário Brasileiro da Gestão do Processo de Projecto na Construção de Edifícios - NUTAU, São Paulo, 2006. Disponível em: $<$ https://repositorium.sdum.uminho.pt/bitstream/ 1822/6792/1/095NUTAU.pdf > . Acesso em: 07 ago. 2016.

John, V. M.; Sato, N. M. N.; Agopyan, V.; Sjöström, C. Durabilidade e sustentabilidade: desafios para a construção civil brasileira. Anais do Workshop sobre durabilidade das construções. São José dos Campos, 2002.

Kibert, C. J. Establishing principles and a model for sustainable construction. Anais do XVI CIB TG 16, Sustainable Construction, Tampa, Florida, USA, 1994. Disponível em: <https://www.irbnet.de/daten/iconda/CIB_DC2 4773.pdf>. Acesso em: 07 ago. 2016.

Mariano, L. S. Gerenciamento de resíduos da construção civil com reaproveitamento estrutural: estudo de caso de uma obra com $\mathbf{4 . 0 0 0 m ^ { 2 }}$. Curitiba: UFPR, 2008. (Dissertação de mestrado) Disponível em: $<$ http://www.ppgerha.ufpr.br/ publicacoes/dissertacoes/files/147-Leila_ Seleme_Mariano.pdf $>$. Acesso em: 30 ago. 2016.

Mateus, R.; Bragança, L. Avaliação da sustentabilidade da construção: desenvolvimento de uma metodologia para a avaliação da sustentabilidade de soluções construtivas. Anais do I Congresso sobre Construção Sustentável, Leça da Palmeira, Portugal, 2004. Disponível em: <http://hdl.handle.net/1822/7333>. Acesso em: 30 maio 2016.
Mateus, R.; Fernandes, J. E. P.; Bragança, L.; Almeida, M. G.; Silva, S. M.; Mendonça, P.; Gervásio, H. (Eds.). Livro de Atas do Seminário reVer 2015: contributos da arquitetura vernácula portuguesa para a sustentabilidade do ambiente construído. Porto, $2015 . \quad$ Disponível em: $<$ http://repositorium.sdum.uminho.pt/handle/18 22/35978>. Acesso em: 30 maio 2016.

Mendonça, P. Vantagens da utilização de materiais naturais ou pouco transformados. Livro de Atas do Seminário reVer, Porto, 2015. Disponível em: $<$ http://repositorium.sdum.uminho.pt/handle/18 22/35978>. Acesso em: 30 maio 2016.

Pinheiro, M. D. Construção sustentável - mito ou realidade? Anais do VII Congresso Nacional de Engenharia do Ambiente, Lisboa, 2003. Disponível em: <https://fenix.tecnico.ulisboa.pt/ downloadFile/3779571242058/PaperAPEA_Co nstrucaoSustentavel.pdf $>$. Acesso em: 30 maio 2016.

Rocheta, V.; Farinha, F. Práticas de projecto e construtivas para a construção sustentável. Anais do III Congresso Nacional, Coimbra, Portugal, Universidade de Coimbra. Disponível em: <http://www.altercexa.eu/test/images/ archivos/2-ROCPra.pdf>. Acesso em: 22 ago. 2016.

Silva Mateus, R. F. M. Avaliação da sustentabilidade da construção: propostas para o desenvolvimento de edifícios mais sustentáveis. Minho: Universidade do Minho, Escola de Engenharia, 2009. (Tese de Doutorado). Disponível em: $<$ http://hdl.handle.net/1822/9886>. Acesso em: 30 maio 2016.

Solano, R. B. P. A importância da arquitetura sustentável na redução do impacto ambiental. Anais do Espaço Sustentável: Inovações em Edifícios e Cidades - NUTAU 2008. Disponível em: <http://www.usp.br/nutau/CD/28.pdf>. Acesso em: 22 ago. 2016.

Torgal, F. P.; Jalali, S. A sustentabilidade dos materiais de construção. 2. ed. Minho: TecMinho, 2010. Disponível em: <http://hdl.handle.net/1822/28852>. Acesso em: 30 maio 2016.

UNEP. Buildings and climate change: status, challenges, and opportunities. Paris: United Nations Environmental Programme, 2007. Disponível em: <http://www.unep.fr/shared/publications/pdf/D TIx0916xPA-BuildingsClimate.pdf $>$. Acesso em: 05 ago. 2016. 
Wieczynski, V. J. Construções mais sustentáveis: alternativas para uma habitação de baixo custo econômico. 2015. Disponível em: $<$ http://www.uniedu.sed.sc.gov.br/wpcontent/uploads/2015/02/Artigo-Vlademir-JoséWieczynski.pdf>. Acesso em: 08 ago. 2016.

Informação da Licença: Este é um artigo Open Access distribuído sob os termos da Licença Creative Commons Attribution, que permite uso irrestrito, distribuição e reprodução em qualquer meio, desde que a obra original seja devidamente citada.

Rev. Bras. Gest. Amb. Sustent., 2016, v. 3, n. 6, p. 255-263. 